\title{
Radio Resource Allocation and Mobility Accessment of 5G Wireless Network Using Adaptive Q-LEACH Routing Protocol
}

\author{
Premi.A ${ }^{\mathrm{a}, 1}$ and Rajakumar. $\mathrm{S}^{\mathrm{b}}$ \\ ${ }^{a} P G$ Scholar, Dept of ECE, Panimalar Engineering College, Chennai, India \\ ${ }^{\mathrm{b}}$ Professor, Dept of ECE, Panimalar Engineering College, Chennai, India
}

\begin{abstract}
The implementation and operation of a Fifth Generation (5G) network aims to achieve a maximum speed, low potential, improved flexibility, and a change in requirements and technologies from service-oriented to user-oriented. The users need resource allocation and management that is effective. Established networks' closed infrastructure and ossified services result in particularly in wireless networks, inefficient resource allocation and underutilized network resources. On the basis the standard of a service provider's utility benefit then customer gratification, various allocation strategies are suggested. Wireless system based $5 \mathrm{G}$ another research area aimed at supply distribution and $5 \mathrm{G}$ access links is network. In this project, radio resource allocation and mobility assessment of $5 \mathrm{G}$ wireless network LEACH routing protocol is implemented. In terms of wireless networks, various architectural integrations of other wireless technologies such as 5G, LTE, Wi-MAX, and so on are highlighted. Furthermore, the project focuses on resource allocation approaches and strategies for cellular networks, as well as comprehensive criteria for future $5 \mathrm{G}$ networks
\end{abstract}

Keywords. High Throughput, LEACH Protocol, Wireless Network, Allocation Techniques.

\section{Introduction}

IP networking is sprouting in current wireless technology to offer a quicker Link to the internet, multimedia software, and a plethora on-demand provision of resources to end users. The launch the $5 \mathrm{G}$ network has resulted in a 1000-fold increase in data rate demand. Currently, 4G provides optimized output for IP-based networks up to $1 \mathrm{Gbps}$. However, there are billions of connected devices on the market today and increasing demand and variety, and a large a large amount of data, a short latency, and a high throughput are needed. The 5G technology of the future is expected to fulfill these needs, and it is pushing new high-definition networking requests and skills in a variety of areas, including the Internet of Things (IoT). The 5G wireless network is usercentric, necessitating efficient resource distribution to meet Quality of Service requirements (QoS). However, with the growing demand for the $5 \mathrm{G}$ wireless network, effective resource distribution is a major challenge.

${ }^{1}$ Premi.A, PG Scholar, Dept of ECE, Panimalar Engineering College, Chennai, India,

E-mail:jackantopremi@gmail.com. 
The $5 \mathrm{G}$ network focuses on spectrum sharing in a network with a lot of diversity and a lot of transmission speeds systems (MIMO).

\section{Related Works}

Marina Dupcinov et.al [1] has for AODV The Improved Neighbor Detection Algorithm Routing Protocol was proposed and here the observed problem is has an effect on the AODV routing protocol's algorithm is decline the Ad hoc network efficiency is affected. Effectively improves data throughput using signal to noise ratio (SNR) value. Tuba Firdaus et.al [2] presented the aim of passive optical network (PON) technology is to provide end-users with a low-cost, interference-free, and bandwidth-abundant access system. The observed problem is based on mobility characteristic of node present in MANET. AlyM.El-Semary et.al [3] has designed BP-AODV: Chaotic Mapbased AODV Routing Protocol with Blackhole Security for MANETs. It cannot resist the cooperative black-hole Two nodes are targeted. AODV and SAODV are not participating together. Rahim Khan et.al [4] presents concerns related to short-range wireless technology for next-generation personal area networks in terms of standardization, regulation, and growth (PAN). Ultra-wideband (UWB) and $60 \mathrm{GHz}$ millimeter-wave networking technologies offer unrivalled short-range wireless broadbandconnectivityandareforerunnersomulti-gigabitwirelessnetworks.

RobbM.Winter et.al [5] designed Enhanced AODV This Incorrect billing and estimations of power consumption may occur as a result of using this unit. Yang Hui et.al [6] Researched Based on a Wireless Mesh Network and the DSDV Routing Protocol.The protocol can provide better delivery rates and lower network latency, however, there is still a serious problem in the network and cluster header load is too heavy. Manjunath $M$ et.al [7] discussed Spatial DSDV (S-DSDV) reduces the misguiding impact of pheromones on unrelated paths by broadcasting routing tables to immediate neighbours with a lower sequence number.

\section{AdaptiveQ-LEACH}

The various types of LEACH protocols are implemented in the proposed system. Out of all LEACH protocols adaptive Q-LEACH is more advanced, the following advantages are gained in the adaptive Q-LEACH protocol. Since the adaptive QLEACH network is divided into sub-sectors, the clusters that form within these subsectors are more deterministic. Adaptive Q-LEACH is a routing protocol that balances the benefits of both location-based and hierarchical-based routing protocols. The restricted flooding term is used, in which packets are transmitted by nodes closer to the destination or in a promoting environment. Individual nodes measure distance and forwarding zone information to determine their progress toward their destination 


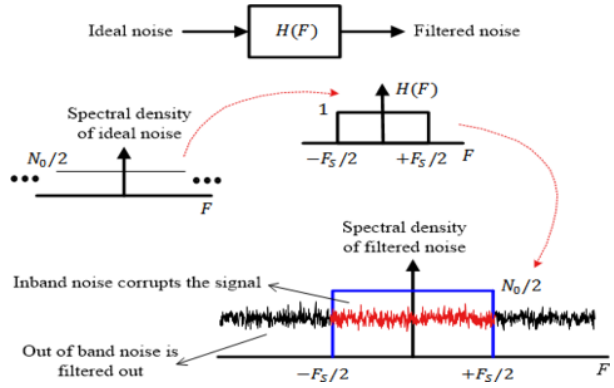

Figure 1.Adaptive Q-LEACH

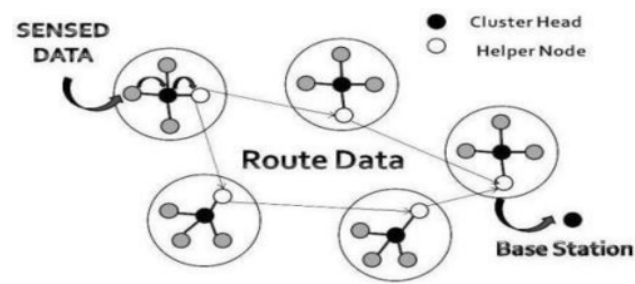

Figure 2.Protocol Model

\section{Fading Channel}

The differences in channel intensity over time and frequency are a defining feature of the mobile wireless channel. The variations can be roughly divided into two types:

1) Signal path loss as a function of distance and shadowing by large objects like buildings and hills cause large-scale fading. This happens when the mobile travels a distance on the order of the cell size, and it is normally frequency agnostic.

2) According to the positive and disruptive interference of the various signal paths between the transmitter and receiver, small-scale fading occurs. This happens on the same spatial scale as the fading forms discussed in this chapter, but with a stronger focus on the latter. Fading on a wide scale is more applicable to problems like cell-siteplanning.

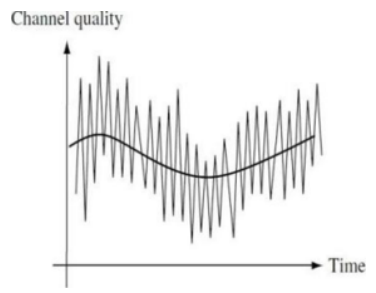

Figure 3. The performance of a channel varies over time 


\section{Result andDiscussion}

Thus in adaptive Q LEACH mobile nodes are dynamically connected. Battery power in the network operating time is determined by mobile nodes adaptive Q-LEACH. So, in addition to reliability and delivery time, energy-conscious routing is a critical aspect to consider. There are two types of energy efficient solutions.

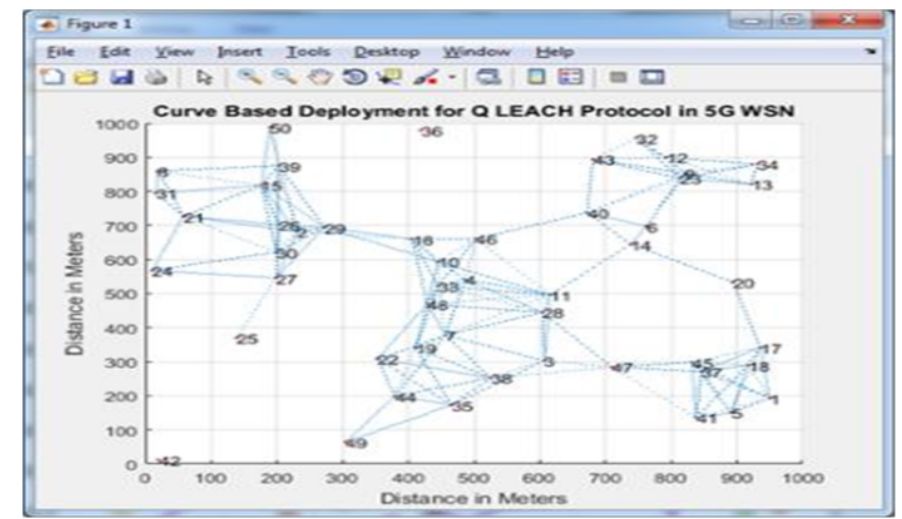

Figure 4.Adaptive Q LEACH protocol clustering

Minimizing the amount of resources needed to send and receivepackets.To prevent needless heeding, a mobile node's idle time should be kept minimum. The adaptive $\mathrm{Q}$ LEACH protocol with clustering of data. The network area covered by the adaptive $Q$ LEACH and residual energy based method. This shows the need of dynamic deployment of the sensor nodes. In both we have deployed the nodes for area $100 \times 100$ but due to adaptive Q LEACH nodes are within the specified area and all nodes will dies in that area only. But in our proposed method the nodes crossed the give area that is some nodes are moved above 100. By this we can say that the coverage area isincreased.

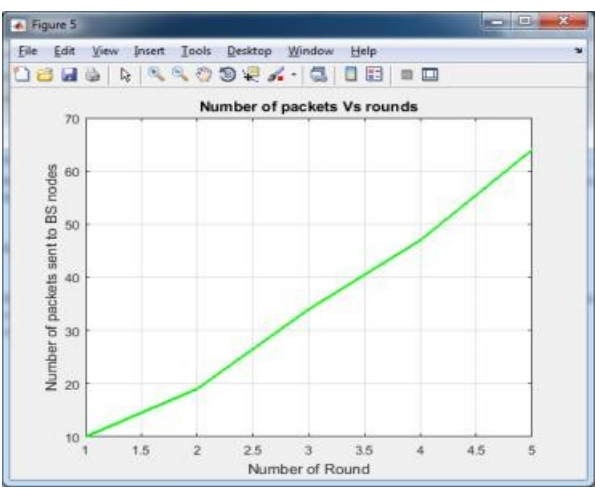

Figure 5.Average energy of eachnodeRound $=50$

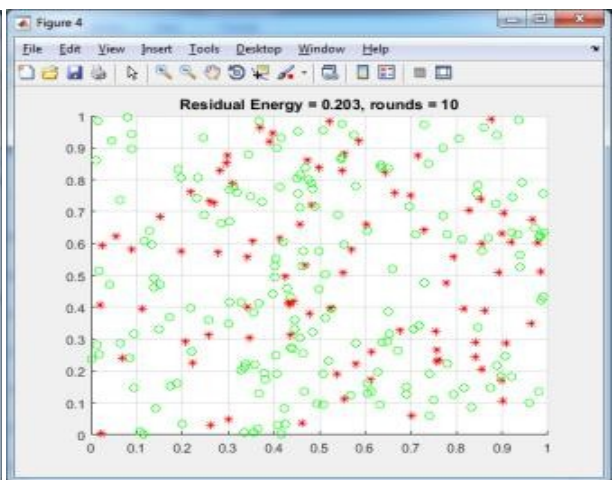

Figure 6. Number of Dead nodesRound $=25$. 
From the graph, we know that there are some nodes that begin to die in about $250 \mathrm{~s}$ of running time adaptive. When all nodes in the Q LEACH protocol died in adaptive mode, the nodes started to die in the 400s. Q LEACH protocol. Here the failure node is node is calculated as zero. The round trip time is used to detect it. Since it takes longer to relay the packet which causes it to be dropped. By comparing the round trip delay time of this path to a threshold value, the failure node is defined.

\section{Conclusion}

For wireless data exchange, wireless network modeling and simulation was used in $5 \mathrm{G}$ environment using adaptive Q-LEACH protocol. In wireless communication, data mobility is enhanced by increasing the SNR and increasing the system's throughput. The BER parameters are used to prevent the ongoing call from being dropped by holding handoff and the possibility of establishing a new call from being blocked due to a temporary lack of an idle channel. By correctly detecting interference, the $5 \mathrm{G}$ wireless network is used to provide safe communication. Calculating different parameters to show the correct implementation of the proposed method enhancesQoS.

\section{References}

[1] Marina Dupcinov. Improved Neighbor Detection Algorithm For AODV Routing Protocol. IEEE CommunicationLetters, VOL.7, No12, December2003.

[2] Tuba Firdauis .Congestion Aware Routing In AODV Based Mobile Ad-Hoc Network (Manet). IEEE J. Sel. Areas Commun s, Vol. 23, No. 2, pp.201-220,2019.

[3] AlyM.El-Semary, BP-AODV: Blackhole Protected AODV Routing Protocol for MANETs based on Chaotic Map. IEEE Trans. Network Commun., vol. 10, no. 11, pp. 3666-3674, Nov.2011.

[4] Rahim Khan .Mac-AODV Based Mutual Authentication Scheme For Constraint Oriented Networks. IEEE Journal of Communications and Networks, vol. 8, pp. 44459-44469,2020.

[5] Yang Hui. Research on DSDV Routing Protocol Based on Wireless Mesh Network. vol. 8 978-1-53861243-9/18/\$31.00 c IEEE, The 30th Chinese Control and Decision (2018CCD).

[6] Manjunath M.Spatial DSDV (S-DSDV) routing algorithm for mobile ad hoc network. Journal on Contemporary Computing and Informatics(IC3I),2014.

[7] Shuo Wu.An Adaptive on-Demand Multipath Routing Protocol With QoS Support for High-Speed MANET. in proc. IEEE Transactions on VéhiculaTechnologie Volume: 8 Journal Article2020.

[8] Margam K. Suthar .Performance Analysis of AODV and DSR Routing Protocol for Wireless AdhocNetwork .International JournalonElectronicsandElectricalEngineering,vol.5,pp.284,2017.

[9] Ould-Khaoua.M . Bandwith-Efficient Routing Protocol For Mobile Ad Hoc Networks. In Proc. IEEE Access Volume: 150, Issue:4 Journal Article,2003. 